\title{
A FUNCTION SPACE TRIPLE OF A COMPACT POLYHEDRON INTO AN OPEN SET IN EUCLIDEAN SPACE
}

\author{
KATSURO SAKAI
}

(Communicated by James E. West)

\begin{abstract}
Let $X$ be a non-zero dimensional compact Euclidean polyhedron and $Y$ an open set in Euclidean space $\mathbf{R}^{r} \quad(r>0)$. The spaces of (continuous) maps, Lipschitz maps and PL maps from $X$ to $Y$ are denoted by $C(X, Y)$, $\operatorname{LIP}(X, Y)$ and $\operatorname{PL}(X, Y)$, respectively. We prove that the triple
\end{abstract}

$$
(C(X, Y), \operatorname{LIP}(X, Y), \operatorname{PL}(X, Y))
$$

is an $(s, \Sigma, \sigma)$-manifold triple, where $s=(-1,1)^{\omega}$,

$$
\begin{gathered}
\Sigma=\{x \in s|\sup | x(i) \mid<1\} \text { and } \\
\sigma=\{x \in s \mid x(i)=0 \text { except for finitely many } i\} .
\end{gathered}
$$

\section{INTRODUCTION}

A paracompact (topological) manifold modelled on a given space $E$ is called an $E$-manifold. For $F \subset E$, an $(E, F)$-manifold pair is a pair $(M, N)$ of an $E$-manifold $M$ and an $F$-manifold $N$ which admits an open cover $\mathscr{U}$ of $M$ and open embeddings $\varphi_{U}: U \rightarrow E, U \in \mathscr{U}$, such that $\varphi_{U}(U \cap N)=\varphi_{U}(U) \cap F$. For $G \subset F \subset E$, an $(E, F, G)$-manifold triple is defined by the same way. Let $Q$ denote the Hilbert cube $[-1,1]^{\omega}, s=(-1,1)^{\omega}$ the pseudo-interior of $Q$,

$$
\begin{gathered}
\Sigma=\{x \in s|\sup | x(i) \mid<1\} \text { and } \\
\sigma=\{x \in s \mid x(i)=0 \text { except for finitely many } i\},
\end{gathered}
$$

where $x(i)$ denotes the $i$-th coordinate of $x$. Note that $s$ is homeomorphic to $(\cong)$ the Hilbert space $\ell_{2}[\mathrm{An}]$. Moreover it is proved in $\left[\mathrm{SW}_{2}\right]$ that $(s, \Sigma, \sigma) \cong$ $\left(\ell_{2}, \ell_{2}^{Q}, \ell_{2}^{f}\right)$, where $\ell_{2}^{Q}$ is the linear span of the Hilbert cube $\prod_{i \in \mathrm{N}}\left[-i^{-1}, i^{-1}\right]$ in $\ell_{2}$ and $\ell_{2}^{f}$ is the linear span of the usual orthonormal basis of $\ell_{2}$, that is,

$$
\begin{gathered}
\ell_{2}^{Q}=\left\{x \in \ell_{2}|\sup | i \cdot x(i) \mid<\infty\right\} \text { and } \\
\ell_{2}^{f}=\left\{x \in \ell_{2} \mid x(i)=0 \text { except for finitely many } i\right\} .
\end{gathered}
$$

Received by the editors May 28, 1988 and, in revised form April 16, 1989.

1980 Mathematics Subject Classification (1985 Revision). Primary 58D15, 57N20.

Key words and phrases. Space of (continuous) maps, space of Lipschitz maps, space of PL maps, polyhedron, convex set, $(s, \Sigma, \sigma)$-manifold triple. 
Let $X=(X, d)$ be a non-discrete metric compactum and $Y=(Y, \rho)$ be a separable metric space without isolated points. The space of (continuous) maps from $X$ to $Y$ is denoted by $C(X, Y)$. The topology of $C(X, Y)$ is induced by the sup-metric

$$
\rho(f, g)=\sup \{\rho(f(x), g(x)) \mid x \in X\} .
$$

Then it is known that $C(X, Y)$ is an $s$-manifold (i.e., $l_{2}$-manifold) if $Y$ is a complete ANR [ $\left.\mathrm{Ge}_{1}, \mathrm{To}, \mathrm{Sa}_{1}\right]$.

By $\operatorname{LIP}(X, Y)$, we denote the subspace of $C(X, Y)$ consisting of all Lipschitz maps. The Lipschitz constant of $f \in \operatorname{LIP}(X, Y)$ is denoted by $\operatorname{lip} f$, i.e.,

$$
\operatorname{lip} f=\inf \{k \geq 0 \mid \rho(f(x), f(y)) \leq k \cdot d(x, y)\} .
$$

For each $k>0$, let

$$
\begin{gathered}
k-\operatorname{LIP}(X, Y)=\{f \in \operatorname{LIP}(X, Y) \mid \operatorname{lip} f \leq k\} \text { and } \\
\operatorname{LIP}_{k}(X, Y)=\{f \in \operatorname{LIP}(X, Y) \mid \operatorname{lip} f<k\}=\bigcup_{k^{\prime}<k} k^{\prime}-\operatorname{LIP}(X, Y) .
\end{gathered}
$$

Then $(C(X, Y), \operatorname{LIP}(X, Y))$ is an $(s, \Sigma)$-manifold pair if $Y$ is a locally compact, locally convex set in a normed linear space $\left[\mathrm{SW}_{1}\right]$ or a Lipschitz manifold or a Euclidean polyhedron $\left[\mathrm{Sa}_{2}\right]$. For each $k>0$,

$$
\left(k-\operatorname{LIP}(X, Y), \operatorname{LIP}_{k}(X, Y)\right) \cong(Q, \Sigma)
$$

if $Y$ is a compact convex set in a normed linear space [SW $\mathrm{SW}_{1}$.

In case $X$ and $Y$ are polyhedra, Geoghegan [ $\left[\mathrm{Ge}_{2}\right]$ proved that $(C(X, Y)$, $\operatorname{PL}(X, Y))$ is an $(s, \sigma)$-manifold pair, where $\operatorname{PL}(X, Y)$ denotes the subspace of $C(X, Y)$ consisting of all PL maps. It is natural to conjecture that the triple

$$
(C(X, Y), \operatorname{LIP}(X, Y), \operatorname{PL}(X, Y))
$$

is an $(s, \Sigma, \sigma)$-manifold triple. In this paper, we prove this conjecture in the case where $Y$ is an open set in Euclidean space $\mathbf{R}^{r}$ (cf. [SW ${ }_{1}$, Conjecture 3.3]).

Main result. Let $X$ be a non-zero dimensional compact polyhedron and $Y$ an open set in Euclidean space $\mathbf{R}^{r}(r>0)$. Then the triple

$$
(C(X, Y), \operatorname{LIP}(X, Y), \operatorname{PL}(X, Y))
$$

is an $(s, \Sigma, \sigma)$-manifold triple.

This is obtained as a corollary of the following:

0.1. Theorem. Let $X$ be a non-zero dimensional compact polyhedron and $Y$ a non-degenerate locally compact convex set in Euclidean space $\mathbf{R}^{r}$. Then

$$
(C(X, Y), \operatorname{LIP}(X, Y), \operatorname{PL}(X, Y)) \cong(s, \Sigma, \sigma) .
$$




\section{Preliminaries}

A closed set $A$ in a metric space $W=(W, d)$ is a $Z$-set if for each map $f: Q \rightarrow W$ and each $\varepsilon>0$, there is a map $g: Q \rightarrow W \backslash A$ with $d(f, g)<\varepsilon$. A countable union of $Z$-sets in $W$ is called a $Z_{\sigma}$-set. A subset $M$ of $W$ is a cap (or fd-cap) set for $W$ if $M=\bigcup_{n \in \mathbf{N}} M_{n}$, where $M_{1} \subset M_{2} \subset \cdots$ is a tower of (resp. finite-dimensional) compact $Z$-sets in $W$ with the property

(fd-)cap: For each (finite-dimensional) compact set $A$ in $W$, each $\varepsilon>0$ and each $m \in \mathbf{N}$, there is an $n \geq m(\in \mathbf{N})$ and an embedding $h: A \rightarrow M_{n}$ such that $h \mid A \cap M_{m}=$ id and $d(h$, id $)<\varepsilon$.

or (fd-)cap sets, refer to [Ch].

The following characterization of $(s, \Sigma, \sigma)$-(or $(Q, \Sigma, \sigma)$-)manifold triples is given in $\left[\mathrm{SW}_{2}\right]$.

1.1. Theorem. A triple $(W, M, N)$ of spaces is an $(s, \Sigma, \sigma)-($ or $(Q, \Sigma, \sigma)-)$ manifold triple if and only if $W$ is an $s$-(or $Q$-)manifold, and there is a tower $M_{1} \subset M_{2} \subset \cdots$ of compact $Q$-manifolds in $W$ with cap such that $M=$ $\bigcup_{i \in \mathbf{N}} M_{i}$, each $M_{i}$ is a $Z$-set in $M_{i+1}$ and each $M_{i} \cap N$ is an fd-cap set for $M_{i}$.

A map $f: X \rightarrow Y$ between metric spaces is locally Lipschitz (abbreviated $L I P$ ) if each $x \in X$ has a neighborhood $U$ such that $f \mid U$ is Lipschitz. Notice, each LIP map $f: X \rightarrow Y$ is Lipschitz if $X$ is compact. A metric space $Y$ is an absolute LIP extensor (abbreviated $A L E$ ) if every LIP map $f: A \rightarrow Y$ of a closed set of an arbitrary metric space $X$ has a LIP extension $\tilde{f}: X \rightarrow Y$.

1.2. Lemma. Any compact convex set $Y$ in Euclidean space $\mathbf{R}^{r}$ is an ALE. Proof. By [Wo, Lemma 4], the nearest point retraction $\nu: \mathbf{R}^{r} \rightarrow Y$ defined by

$$
\|x-\nu(x)\|=\operatorname{dist}(x, Y)=\inf \{\|x-y\| \mid y \in Y\}
$$

is Lipschitz, where $\|\cdot\|$ is the Euclidean norm. Since $\mathbf{R}^{r}$ is an ALE by [LV, Theorem 5.7], $Y$ is also an ALE.

\section{LIPSCHITZ CONSTANTS OF PL MAPS}

For each $x \in \mathbf{R}^{n}, x(i)$ denotes the $i$-th coordinate of $x$. The Euclidean norm of $\mathbf{R}^{n}$ is Lipschitz equivalent to the norm defined as follows:

$$
\|x\|=|x(1)|+\cdots+|x(n)| \quad \text { for } x \in \mathbf{R}^{n} .
$$

Let $\left\{\mathbf{e}_{1}, \ldots, \mathbf{e}_{q}\right\}$ be the canonical orthonormal basis for $\mathbf{R}^{q}$, that is, $\mathbf{e}_{i}(j)=0$ if $i \neq j$ and $\mathbf{e}_{i}(i)=1$. The $q$-th symmetric group is denoted by $\Sigma_{q}$. For each $\alpha=(\alpha(1), \cdots, \alpha(q)) \in \Sigma_{q}, \Delta(\alpha)$ denotes the full simplicial complex with vertices

$$
\begin{gathered}
v_{0}^{\alpha}=0, \quad v_{1}^{\alpha}=\mathbf{e}_{\alpha(1)}, \\
v_{2}^{\alpha}=\mathbf{e}_{\alpha(1)}+\mathbf{e}_{\alpha(2)}, \cdots, \quad v_{q}^{\alpha}=\mathbf{e}_{\alpha(1)}+\cdots+\mathbf{e}_{\alpha(q)} .
\end{gathered}
$$


2.1. Lemma. For each linear (affine) map $f:|\Delta(\alpha)| \rightarrow \mathbf{R}$,

$$
\operatorname{lip} f=\max \left\{\left|f\left(v_{i}^{\alpha}\right)-f\left(v_{i-1}^{\alpha}\right)\right| \mid i=1, \ldots, q\right\} .
$$

Proof. First observe that for each $x \in|\Delta(\alpha)|$,

$$
1 \geq x(\alpha(1)) \geq x(\alpha(2)) \geq \cdots \geq x(\alpha(q)) \geq 0
$$

and

$$
\begin{aligned}
x= & (1-x(\alpha(1))) \cdot v_{0}^{\alpha}+(x(\alpha(1))-x(\alpha(2))) \cdot v_{1}^{\alpha}+\cdots \\
& +(x(\alpha(q-1))-x(\alpha(q))) \cdot v_{q-1}^{\alpha}+x(\alpha(q)) \cdot v_{q}^{\alpha}
\end{aligned}
$$

Since $f$ is linear, it follows that

$$
\begin{aligned}
f(x)= & (1-x(\alpha(1))) \cdot f\left(v_{0}^{\alpha}\right)+(x(\alpha(1))-x(\alpha(2))) \cdot f\left(v_{1}^{\alpha}\right)+\cdots \\
& +(x(\alpha(q-1))-x(\alpha(q))) \cdot f\left(v_{q-1}^{\alpha}\right)+x(\alpha(q)) \cdot f\left(v_{q}^{\alpha}\right) .
\end{aligned}
$$

Then for each $x, x^{\prime} \in|\Delta(\alpha)|$,

$$
\begin{aligned}
\left|f(x)-f\left(x^{\prime}\right)\right| \leq & \left|x(\alpha(1))-x^{\prime}(\alpha(1))\right| \cdot\left|f\left(v_{1}^{\alpha}\right)-f\left(v_{0}^{\alpha}\right)\right|+\cdots \\
& +\left|x(\alpha(q))-x^{\prime}(\alpha(q))\right| \cdot\left|f\left(v_{q}^{\alpha}\right)-f\left(v_{q-1}^{\alpha}\right)\right| \\
\leq & \left\|x-x^{\prime}\right\| \cdot \max \left\{\left|f\left(v_{i}^{\alpha}\right)-f\left(v_{i-1}^{\alpha}\right)\right| \mid i=1, \ldots, q\right\} .
\end{aligned}
$$

Thus we have the result.

For each $c>0$ and $v \in \mathbf{R}^{q}$, let $h_{c, v}: \mathbf{R}^{q} \rightarrow \mathbf{R}^{q}$ be the (affine) homeomorphism defined by $h_{c, v}(x)=c \cdot x+v$. For each $p \in \mathbf{N}$, we define the triangulation $K(q, p)$ of $I^{q}=[0,1]^{q}$ as follows:

$$
K(q, p)=\left\{h_{1 / p, v}(\sigma) \mid \sigma \in \Delta(\alpha), \alpha \in \Sigma_{q}, v \in V_{p}^{q}\right\},
$$

where

$$
V_{p}^{q}=\left\{0, \frac{1}{p}, \ldots, \frac{p-1}{p}\right\}^{q} \subset I^{q} .
$$

2.2. Lemma. Let $f: I^{q} \rightarrow \mathbf{R}$ be a map such that $f \mid \sigma$ is linear for each $\sigma \in$ $K(q, p)$. Then

$$
\operatorname{lip} f=\operatorname{lip}\left(f \mid K(q, p)^{0}\right) .
$$

Proof. Let $k=\operatorname{lip}\left(f \mid K(q, p)^{0}\right)$. It suffices to show that $\operatorname{lip} f \leq k$. First we show that $\operatorname{lip}(f \mid \sigma) \leq k$ for each $\sigma \in K(q, p)$. Choose an $\alpha \in \Sigma_{q}$ and $v \in V_{p}^{q}$ so that $h_{1 / p, v}^{-1}(\sigma) \in \Delta(\alpha)$. Observe that $\operatorname{lip}\left(h_{1 / p, v}^{-1}\right)=p$ and

$$
\left\|h_{1 / p, v}\left(v_{i}^{\alpha}\right)-h_{1 / p, v}\left(v_{i-1}^{\alpha}\right)\right\|=\frac{1}{p}
$$

for each $i=1, \ldots, q$. By Lemma 2.1, we have

$$
\begin{aligned}
\operatorname{lip}(f \mid \sigma) & \leq \operatorname{lip}\left(f \circ h_{1 / p, v} \| \Delta(\alpha) \mid\right) \cdot \operatorname{lip}\left(h_{1 / p, v}^{-1} \mid \sigma\right) \\
& \leq p \cdot \max \left\{\mid f h_{1 / p, v}\left(v_{i}^{\alpha}\right)-f h_{1 / p, v}\left(v_{i-1}^{\alpha}\right) \| i=1, \ldots, q\right\} \\
& \leq p \cdot \max \left\{k \cdot\left\|h_{1 / p, v}\left(v_{i}^{\alpha}\right)-h_{1 / p, v}\left(v_{i-1}^{\alpha}\right)\right\| \mid i=1, \ldots, q\right\} \\
& =k .
\end{aligned}
$$


Now we show $\operatorname{lip} f \leq k$. Let $x \neq x^{\prime} \in I^{q}=|K(q, p)|$. If $x$ and $x^{\prime}$ are contained in same $\sigma \in K(q, p)$, then

$$
\left|f(x)-f\left(x^{\prime}\right)\right| \leq \operatorname{lip}(f \mid \sigma) \cdot\left\|x-x^{\prime}\right\| \leq k \cdot\left\|x-x^{\prime}\right\| .
$$

Otherwise choose points $x_{0}, x_{1}, \ldots, x_{n}$ on the straight line segment connecting $x$ and $x^{\prime}$ so that $x_{0}=x, x_{n}=x^{\prime}$, and each pair $x_{i-1}$ and $x_{i}$ are contained in some $\sigma_{i} \in K(q, p)$. Since

$$
\left\|x-x^{\prime}\right\|=\left\|x_{0}-x_{1}\right\|+\left\|x_{1}-x_{2}\right\|+\cdots+\left\|x_{n-1}-x_{n}\right\|,
$$

we have

$$
\begin{aligned}
\left|f(x)-f\left(x^{\prime}\right)\right| & \leq\left|f\left(x_{0}\right)-f\left(x_{1}\right)\right|+\cdots+\left|f\left(x_{n-1}\right)-f\left(x_{n}\right)\right| \\
& \leq \operatorname{lip}\left(f \mid \sigma_{1}\right) \cdot\left\|x_{0}-x_{1}\right\|+\cdots+\operatorname{lip}\left(f \mid \sigma_{n}\right) \cdot\left\|x_{n-1}-x_{n}\right\| \\
& \leq k \cdot\left\|x-x^{\prime}\right\| .
\end{aligned}
$$

This completes the proof.

2.3. Corollary. Let $f: I^{q} \rightarrow \mathbf{R}^{r}$ be a map such that $f \mid \sigma$ is linear for each $\sigma \in K(q, p)$. Then

$$
\operatorname{lip} f \leq r \cdot \operatorname{lip}\left(f \mid K(q, p)^{0}\right) .
$$

Proof. For each $i=1, \ldots, r$, let $\pi_{i}: \mathbf{R}^{r} \rightarrow \mathbf{R}$ denote the projection onto the $i$-th coordinates. By Lemma 2.2,

$$
\begin{aligned}
\operatorname{lip} \pi_{i} f & =\operatorname{lip}\left(\pi_{i} f \mid K(q, p)^{0}\right) \\
& \leq\left(\operatorname{lip} \pi_{i}\right) \cdot\left(\operatorname{lip}\left(f \mid K(q, p)^{0}\right)\right) \\
& \leq \operatorname{lip}\left(f \mid K(q, p)^{0}\right) .
\end{aligned}
$$

Then for each $x, x^{\prime} \in I^{q}$,

$$
\begin{aligned}
\left\|f(x)-f\left(x^{\prime}\right)\right\| & =\left|\pi_{1} f(x)-\pi_{1} f\left(x^{\prime}\right)\right|+\cdots+\left|\pi_{r} f(x)-\pi_{r} f\left(x^{\prime}\right)\right| \\
& \leq\left(\operatorname{lip} \pi_{1} f+\cdots+\operatorname{lip} \pi_{r} f\right) \cdot\left\|x-x^{\prime}\right\| \\
& \leq r \cdot \operatorname{lip}\left(f \mid K(q, p)^{0}\right) \cdot\left\|x-x^{\prime}\right\| . \quad \square
\end{aligned}
$$

2.4. Lemma. Let $Y$ be a convex set in Euclidean space $\mathbf{R}^{r}$. Then for each $q \in \mathbf{N}, k>0$ and $\varepsilon>0$, there is a map

$$
\varphi: k-\operatorname{LIP}\left(I^{q}, Y\right) \rightarrow \operatorname{PL}\left(I^{q}, Y\right) \cap r k-\operatorname{LIP}\left(I^{q}, Y\right)
$$

which is $\varepsilon$-close to id.

Proof. Choose $p \in \mathbf{N}$ so large that

$$
\operatorname{mesh} K(q, p)<\varepsilon /(r+1) k \text {. }
$$

Since $Y$ is convex, the desired map $\varphi$ can be defined as follows:

$$
\varphi(f)\left|K(q, p)^{0}=f\right| K(q, p)^{0}
$$

and

$$
\varphi(f) \mid \sigma \text { is linear for each } \sigma \in K(q, p) \text {. }
$$


In fact, $\operatorname{lip} \varphi(f) \leq r \cdot \operatorname{lip} f \leq r k$ by the above corollary. For each $x \in I^{q}$, we have a $v \in K(q, p)^{0}$ such that $\|x-v\|<\varepsilon /(r+1) k$. Then

$$
\begin{aligned}
\|\varphi(f)(x)-f(x)\| & \leq\|\varphi(f)(x)-\varphi(f)(v)\|+\|f(x)-f(v)\| \\
& \leq r k \cdot\|x-v\|+k \cdot\|x-v\| \\
& \leq \varepsilon .
\end{aligned}
$$

Thus $\varphi$ is $\varepsilon$-close to id.

\section{SPACE OF $k$-LiPSChitz PL MAPS}

Let $X$ be a non-zero dimensional compact polyhedron in Euclidean space $\mathbf{R}^{q}$ and $Y$ a non-degenerate locally compact convex set in Euclidean space $\mathbf{R}^{r}$. Then we can use the metrics for $X$ and $Y$ defined by the norm in $\S 2$ instead of Euclidean norm to prove the theorem. This norm is of great advantage to our problem as seen in Section 2 and since the map $\|\cdot\|: \mathbf{R}^{q} \rightarrow \mathbf{R}$ is PL. Let us regard $C(X, Y)$ as a subset of the Banach space $C\left(X, \mathbf{R}^{r}\right)$ with the sup-norm

$$
\|f\|=\sup \{\|f(x)\| \mid x \in X\} .
$$

Then note that $\operatorname{PL}(X, Y) \subset C(X, Y)$ and each $k-\operatorname{LIP}(X, Y)$ are convex sets in $C\left(X, \mathbf{R}^{r}\right)$.

3.1. Lemma. For each $k>0, \operatorname{PL}(X, Y) \cap k-\operatorname{LIP}(X, Y)$ is infinite dimensional.

Proof. Without loss of generality, we may assume $0 \in Y$. Let $K$ be a triangulation of $X$ and $v_{0} \in K^{0}$ be a non-isolated vertex. For each $n \in \mathbf{N}$, choose any $v_{n} \in \operatorname{Lk}\left(v_{0}, \operatorname{Sd}^{n} K\right)^{0}$, where $\operatorname{Sd}^{n} K$ is the $n$-th barycentric subdivision of $K$. Let $g_{n} \in \operatorname{PL}(X, Y)$ so that $g_{n}(v)=0$ for $v \in\left(\operatorname{Sd}^{n} K\right)^{0} \backslash\left\{v_{n}\right\}, g_{n}\left(v_{n}\right) \neq 0$ and $g_{n} \mid \sigma$ is linear for each $\sigma \in \operatorname{Sd}^{n} K$. By choosing $g_{n}\left(v_{n}\right)$ close to 0 , we have

$$
g_{n} \in \mathrm{PL}(X, Y) \cap k-\operatorname{LIP}(X, Y) .
$$

It is easy to verify that $\left\{g_{n}\right\}_{n \in \mathbf{N}}$ are linearly independent. Thus $\operatorname{PL}(X, Y) \cap$ $k$ - $\operatorname{LIP}(X, Y)$ is infinite-dimensional.

By [Ge ${ }_{2}$, Lemma 4.3], $\operatorname{PL}(X, Y)$ is $\sigma$-fd-compact, that is, a countable union of finite-dimensional compacta. Then $\operatorname{PL}(X, Y) \cap k-\operatorname{LIP}(X, Y)$ is an infinitedimensional $\sigma$-fd-compact convex set in the Banach space $C\left(X, \mathbf{R}^{r}\right)$. If $Y$ is compact,

$$
\begin{aligned}
& \operatorname{cl}_{C\left(X, \mathbf{R}^{r}\right)}(\operatorname{PL}(X, Y) \cap k-\operatorname{LIP}(X, Y)) \\
& \quad=\mathrm{cl}_{C(X, Y)}(\operatorname{PL}(X, Y) \cap k-\operatorname{LIP}(X, Y)) \subset k-\operatorname{LIP}(X, Y),
\end{aligned}
$$

which are compact by Arzela-Ascoli's Theorem. By [Do, Theorem 2], we have the following theorem. 
3.2. Theorem. If $Y$ is compact, then, for each $k>0$,

$$
\left(\mathrm{cl}_{C(X, Y)}(\mathrm{PL}(X, Y) \cap k-\operatorname{LIP}(X, Y)), \operatorname{PL}(X, Y) \cap k-\operatorname{LIP}(X, Y)\right) \cong(Q, \sigma) .
$$

In the above, it is natural to ask whether or not

$$
\mathrm{cl}_{C(X, Y)}(\mathrm{PL}(X, Y) \cap k-\operatorname{LIP}(X, Y))=k-\operatorname{LIP}(X, Y) .
$$

The author has not succeeded in giving its proof nor a counterexample. This is related to Problem 4.1.

The following lemma follows from the proof of [ $\mathrm{SW}_{1}$, Lemma 1.2]:

3.3. Lemma. For each $k>0$ and $\varepsilon>0$, there exists a map

$$
\psi: k-\operatorname{LIP}(X, Y) \rightarrow k-\operatorname{LIP}(X, Y) \backslash \operatorname{LIP}_{k}(X, Y)
$$

such that $\psi$ is $\varepsilon$-close to id and

$$
\psi(\operatorname{PL}(X, Y) \cap k-\operatorname{LIP}(X, Y)) \subset \operatorname{PL}(X, Y) \cap k-\operatorname{LIP}(X, Y) .
$$

Proof. In fact, we use the norm $\|\cdot\|$ in $\S 2$ which is PL. Then it is easily observed that the map $\psi$ defined in $\left[\mathrm{SW}_{1}\right.$, Lemma 1.2] maps $\operatorname{PL}(X, Y) \cap k-\operatorname{LIP}(X, Y)$ into itself.

3.4. Lemma. For each compact set $A \subset C(X, Y)$ and $\varepsilon>0$, there is a $k>0$ and a map

$$
\varphi: A \rightarrow \operatorname{PL}(X, Y) \cap k-\operatorname{LIP}(X, Y)
$$

which is $\varepsilon$-close to id.

Proof. Without loss of generality, we can assume that $X \subset I^{q}$. Let $R$ : $C\left(I^{q}, Y\right) \rightarrow C(X, Y)$ be the restriction map, that is, $R(f)=f \mid X$ for each $f \in C\left(I^{q}, Y\right)$. Note that for any $k>0$,

$$
R\left(\operatorname{PL}\left(I^{q}, Y\right) \cap k-\operatorname{LIP}\left(I^{q}, Y\right)\right) \subset \operatorname{PL}(X, Y) \cap k-\operatorname{LIP}(X, Y) .
$$

Since $Y$ is an AR, there is an extension map $E: C(X, Y) \rightarrow C\left(I^{q}, Y\right)$, namely a right inverse of $R$, i.e., $R \circ E=$ id. In fact, the evaluation $e: X \times C(X, Y) \rightarrow$ $Y$ extends to a map $\tilde{e}: I^{q} \times C(X, Y) \rightarrow Y$. Then $E$ can be defined by $E(f)(x)=\tilde{e}(x, f)$. By [SW 1 , Lemma 1.4], we have a $k^{\prime}>0$ and a map $\varphi^{\prime}: E(A) \rightarrow k^{\prime}$-LIP $\left(I^{q}, Y\right)$ which is $\varepsilon / 2$-close to id. Let $k=r k^{\prime}>0$. By Lemma 2.4 , we have a map

$$
\psi: k^{\prime}-\operatorname{LIP}\left(I^{q}, Y\right) \rightarrow \operatorname{PL}\left(I^{q}, Y\right) \cap k-\operatorname{LIP}\left(I^{q}, Y\right)
$$

which is $\varepsilon / 2$-close to id. Then the composition

$$
\varphi=R \circ \psi \circ \varphi^{\prime} \circ E: A \rightarrow \operatorname{PL}(X, Y) \cap k-\operatorname{LIP}(X, Y)
$$

is the desired map. In fact, for each $f \in A$ and $x \in X$,

$$
\begin{aligned}
\|\varphi(f)(x)-f(x)\|= & \left\|\psi\left(\varphi^{\prime}(E(f))\right)(x)-E(f)(x)\right\| \\
\leq & \left\|\psi\left(\varphi^{\prime}(E(f))\right)(x)-\varphi^{\prime}(E(f))(x)\right\| \\
& +\left\|\varphi^{\prime}(E(f))(x)-E(f)(x)\right\| \\
\leq & \|\psi-\mathrm{id}\|+\left\|\varphi^{\prime}-\mathrm{id}\right\|<\varepsilon / 2+\varepsilon / 2=\varepsilon .
\end{aligned}
$$




\section{Proof of Theorem 0.1}

First recall that $C(X, Y) \cong s$. Write $Y=\bigcup_{n \in \mathrm{N}} Y_{n}$, where each $Y_{n}$ is a non-degenerate compact convex set and $Y_{n} \subset$ int $_{Y} Y_{n+1}$. For each $n \in \mathbf{N}$, let

$$
M_{n}=\mathrm{cl}_{C(X, Y)}\left(\mathrm{PL}\left(X, Y_{n}\right) \cap n-\operatorname{LIP}\left(X, Y_{n}\right)\right) \text {. }
$$

It follows from Lemma 3.3 that each $M_{n}$ is a $Z$-set in $M_{n+1}$. And by Theorem 3.2, $M_{n} \cong Q$ and

$$
\operatorname{PL}(X, Y) \cap M_{n}=\operatorname{PL}(X, Y) \cap n-\operatorname{LIP}\left(X, Y_{n}\right)
$$

is an fd-cap set for $M_{n}$.

We will show that $\operatorname{LIP}(X, Y)=\bigcup_{n \in \mathrm{N}} M_{n}$. Without loss of generality, we can assume that $X \subset I^{q}$. Let $f \in \operatorname{LIP}(X, Y)$. From compactness, $f(X) \subset Y_{n^{\prime}}$ for some $n^{\prime} \in \mathbf{N}$. By Lemma 1.2, $f$ has an extension $\tilde{f} \in \operatorname{LIP}\left(I^{q}, Y_{n^{\prime}}\right)$. Let $n \geq \max \left\{n^{\prime}, r \cdot \operatorname{lip} \tilde{f}\right\}$. Then by Lemma 2.4 ,

$$
\tilde{f} \in \mathrm{cl}_{C\left(I^{q}, Y\right)} \operatorname{PL}\left(I^{q}, Y_{n}\right) \cap n-\operatorname{LIP}\left(I^{q}, Y_{n}\right) .
$$

Therefore $f=\tilde{f} \mid X \in M_{n}$.

By using Lemma 3.4, we can prove similarly as [SW $\mathrm{SW}_{1}$, Theorem 2.1] that the tower $\left\{M_{n}\right\}_{n \in \mathrm{N}}$ has the property cap for $C(X, Y)$. Thus Theorem 0.1 follows from Theorem 1.1.

Finally we pose the following problem:

4.1. Problem. If $Y$ is a non-degenerate compact convex set, then for each $k>0$, is

$$
\left(k-\operatorname{LIP}(X, Y), \operatorname{LIP}_{k}(X, Y), \operatorname{PL}(X, Y) \cap \operatorname{LIP}_{k}(X, Y)\right) \cong(Q, \Sigma, \sigma) ?
$$

\section{REFERENCES}

[An] R. D. Anderson, Hilbert space is homeomorphic to the countable infinite product of lines, Bull. Amer. Math. Soc. 72 (1966), 515-519.

[Ch] T. A. Chapman, Dense sigma-compact subsets of infinite-dimensional manifolds, Trans. Amer. Math. Soc. 154 (1971), 399-426.

[Do] T. Dobrowolski, The compact Z-set property in convex sets, Topology Appl. 23 (1986), 163172.

[Ge $\left.\mathrm{G}_{1}\right] \mathrm{R}$. Geoghegan, On spaces of homeomorphisms, embeddings, and functions I, Topology 11 (1972), 159-177.

[Ge $\left.\mathrm{Ge}_{2}\right] \ldots$, On spaces of homeomorphisms, embeddings, and functions, II: The piecewise linear case, Proc. London Math. Soc. (3) 27 (1973), 463-483.

[LV] J. Luukkainen and J. Väisälä, Elements of Lipschitz topology, Ann. Acad. Sci. Fenn. Ser. A. I. Math. 3 (1977), 85-122.

[Sa $\left.{ }_{1}\right]$ K. Sakai, The space of cross-sections of a bundle, Proc. Amer. Math. Soc. 103 (1988), 956960.

[Sa $\left.\mathrm{Sa}_{2}\right]$, The space of Lipschitz maps from a compactum to an absolute neighborhood LIP extensor, preprint.

[SW 1 ] K. Sakai and R. Y. Wong, The space of Lipschitz maps from a compactum to a locally convex set, Topology Appl., 32 (1989), 223-235. 
[SW $\left.\mathrm{SW}_{2}\right]$ _- On infinite-dimensional manifold triples, Trans. Amer. Math. Soc., (to appear).

[To] H. Torunczyk, Concerning locally homotopy negligible sets and characterization of $l_{2}$ manifolds, Fund. Math. 101 (1978), 93-110.

[Wo] R. Y. Wong, Lipschitz conjugation and extension of homeomorphisms in $l_{p}$-spaces, J. Math. Analysis Appl. 32 (1970), 573-583.

Institute of Mathematics, University of Tsukuba, Tsukuba-City, 305 Japan 